\title{
Self calibration of a road stereo color vision system using a similarity criterion
}

\author{
Sebastien Kramm \\ sebastien.kramm@univ-rouen.fr
}

\author{
Abdelaziz Bensrhair \\ abdelaziz.bensrhair@insa-rouen.fr \\ L.I.T.I.S., INSA Rouen - BP 08 \\ F-76131 Mont-Saint-Aignan Cedex \\ France
}

\author{
Pierre Miché \\ pierre.miche@insa-rouen.fr
}

\begin{abstract}
In this article, we address the problem of self calibrating an embedded stereoscopic color vision system, by matching interest points, and computing the related fundamental matrix. We propose a new method based on similarity of image areas surrounding automatically detected interest points. We provide some experimental results, and compare these to those obtained with a classical method.
\end{abstract}

Index Terms - Stereo vision, matching, epipolar geometry, fundamental matrix

\section{INTRODUCTION}

Embedded vision systems will be in the next decade one of the majors enhancements in automobile driving assistance. Among these, stereovision presents the interesting feature of retrieving 3D information. Many labs and industrials have developed algorithms for various tasks, including automatic driving ("Darpa contest"), obstacle detection, or other "precrash" systems (See [1][2][3] for a few examples). Most of these applications rely on an aligned image pair, as this reduces computing cost, and is almost mandatory for realtime applications. This can be obtained either through mechanically-aligned cameras, or by rectifying the image pair, using data obtained in a calibration phase [4].

The first approach is by far simpler, as data doesn't have to be pre-processed, but it is also the most demanding, and therefore the most expensive. It is not suitable for industrial mass-production vision systems, as in common vehicles, it would be difficult to achieve this alignment. Moreover, mechanical vibrations will induce drifting.

The second approach is much more interesting, but it needs calibration data. Camera and stereo vision system calibration has been extensively studied (see [5][6]), but most of the methods rely on an off-line calibration phase, using a calibration pattern, which is quite constraining.

We consider here the on-line approach, where the intrinsic parameters are supposed to be known and stable, and we will focus on automatically extracting the stereo geometry, contained in the well-known Fundamental matrix, using only scene elements. (see some classical works [7] for an introduction on epipolar geometry and stereovision.). This will enable the system to be self-sufficient. Besides its principal task that will remain scene analysis, it will periodically recalibrate itself, using only the current scene elements, allowing to rectify image pairs before processing (see [8][9] for recent works on rectifying).

Stereoscopic calibration has been well studied in the past years, but many papers focus either on intrinsic parameters [10], or on Fundamental matrix properties [6], and leave aside "real-world" implementations. In this paper, we assume that the cameras intrinsic parameters are known.

This article describes our approach of the three steps commonly involved in the self-calibration process, that is:

1. Selecting points of interest (i.e. "corners")

2. Matching them

3. Computing the associated Fundamental matrix

The third point has been well studied, and it is commonly admitted that the most usable algorithms in real-world situations, where outliers are unavoidable, are the LMEDS and the RANSAC algorithms, which we will also use.

The most difficult points is matching. Zhang has published a now classical method [11], based on the assumption that a correct match will be surrounded by other correct matches. He introduces a "Strength of Match" (SM) criterion, that takes into account both the candidate matches correlation score, and the neighbors correlation scores, counterbalanced with their relative distance. Through a relaxation process, this method minimizes the sum of all SM, in order to obtain the optimal subset of correct matches

We experimented this method (see [12]), but it turned out to be deceptive in a road context, where corner density can be very sparse. A lonely match can be completely correct, although it has no neighbors. We introduce here a new approach, based on a simple but efficient idea, using the similarity of areas surrounding corners. Finally, we present some experimental results, and discuss them.

In this paper, we focus only on the algorithmic strategy, and do not take into account possible occlusions, as in a road context, most of the points of interest are located far enough to minimize them. This aspect has been dealt with by other authors [13][14]. Our goal will be here to minimize the numbers of outliers, in order to compute the most accurate fundamental matrix.

\section{SELECTING POINTS OF INTEREST}

The first step of the process consists in extracting interest points (i.e. "corners") in both images, to get some material 
for the further matching algorithm. Only the luminance channel of the image is used, as it contains most of the information. We used here classical corner detectors, the more recent SIFT method [18], while invariant to rotation, gives a much higher number of points, and seems to be much slower, making it unsuitable at the moment for our application.

We experimented the classical Harris corner detector [15], and the "Minimum Eigen Value" method, that searches for the minimum eigenvalue of the derivative covariation matrix $\mathrm{M}$ for every pixel. Both of these methods where experimented, using OpenCv [16] implementation. The latter has given better results on road scenes, it appears to be more sensitive than the Harris detector. These methods give an integer position of the detected corner, refining them to a sub-pixel accuracy has shown to be unreliable. In our implementation, we use a minimum distance of 15 pixels between two corners, and a quality level of 0.04 , which provides between 100 to 200 points per image, on a $640 \times 480$ pixels image.

\section{MATCHING INTEREST POINTS}

This process is split up into two tasks: first, building a list of possible matches, using a distance and a correlation constraint, and second, selecting correct matches in this set, using more tight constraints.

\section{A - Establishing a set of candidate matches}

We establish a list of possible matches, which are called "candidates matches". This list is obtained by parsing, for every left corner, all the right ones: We first consider if the distance between the left corner and the right corner is below a predefined threshold. We use 3 distance criteria, the vertical distance, the horizontal distance, and the euclidean distance, as show on figure 1. This way, the system can be tuned precisely to match the type of geometry and the type of scene.

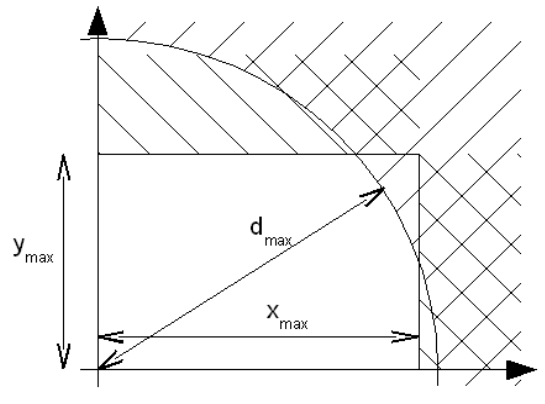

Fig. 1 : maximum matching distance

One must be careful with these settings, as in a typical horizontal camera alignment configuration, close objects can have a high disparity value. Moreover, camera rotation around the roll axis can cause a high vertical disparity on the edges of the image. Thus it is recommended to set these parameters rather loose, as potential bad matches will anyhow be discarded later.

In order to avoid distance problems, we usually limit the useful area to an ROI that only considers the $2 / 3$ of the upper part of the image. In a road context, this allows us to discard the area really close, that has naturally high disparity values.

For all the potential matches, i.e. those for which the distance from left corner to right corner is correct, we then compute each candidate correlation score, using only the luminance information. Two criteria have been used, ZNCC (Zero-mean Normalized Cross Correlation) and ZNSSD (Zero-mean Normalized Sum of Squared Differences). These seem to be the most commonly used in the vision community (See fig. 2 \& 3). We implemented a cooperative method : only the matches giving a score above a threshold for both correlation methods are filed into the candidate matches list.

\begin{tabular}{|l}
$c s_{Z N S S D}=\frac{\sum_{i, j}\left[I_{1}^{\prime}\left(x_{1}+i, y_{1}+j\right)-I_{2}^{\prime}\left(x_{2}+i, y_{2}+j\right)\right]^{2}}{n o r m}$ \\
with : norm $=\sqrt{\sum_{i, j} I_{1}\left(x_{1}+i, y_{1}+j\right)^{2} \sum_{i, j} I_{2}\left(x_{2}+i, y_{2}+j\right)^{2}}$ \\
and : $I_{k}^{\prime}(x+i, y+j)=I_{k}(x+i, y+j)-\bar{I}_{k}\left(x_{k}, y_{k}\right)$ \\
$\bar{I}_{k}(x, y)$ being the mean of the luminance value over the \\
correlation window
\end{tabular}

Fig. 2 : The ZNSSD correlation score

$c s_{Z N C C}=\frac{\sum_{i, j} I_{1}{ }^{\prime}\left(x_{1}+i, y_{1}+j\right) \cdot I_{2}{ }^{\prime}\left(x_{2}+i, y_{2}+j\right)}{n o r m}$

Fig. 3 : The ZNCC correlation score

The first criterion will give 0.0 for identical images, and the second one will give 1.0, and can give negative values. In order to be able to use them in the same algorithms, for the ZNCC criterion, we store the value (1) :

$$
c s=\frac{1-c s_{Z N C C}}{2}
$$

This phase gives a high number of candidate matches, typically between 1000 to 3000 , among which we must now find a subset of correct matches, using an additional unicity constraint, based on neighborhood similarity.

\section{$B$ - The proposed "similarity" method}

The "Zhang" method can be used for color images, but one of its weaknesses is that it doesn't take the color information into account. We propose here another method, that considers the whole image information. The basic idea is 
that, in most cases, in both images, the area surrounding a point will have the same statistical characteristics, mean value and standard deviation.

First, we split both images into 3 color planes: hue, saturation and luminance. Then, around each corner detected in the luminance plane of both images, we extract a small area surrounding the point, and divide it into 9 sub-images (see fig. 4\&5). In our implementation, we use an area of $33 \mathrm{x}$ 33 pixels (must be a multiple of 3 ). The size of the area must be sufficient, as too small areas wouldn't give significant results, and a too large area would increase computational time, not to say about image occlusions that might appear.

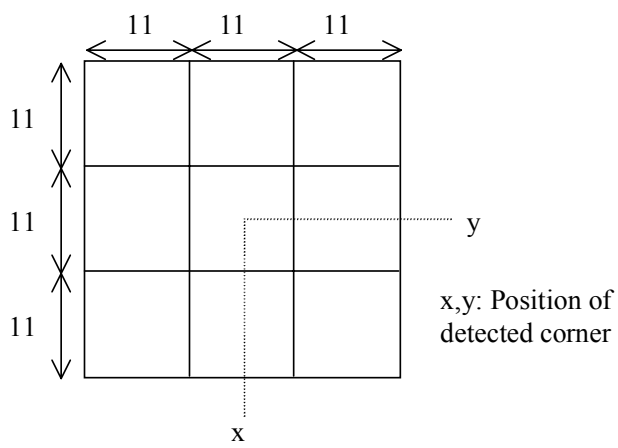

Fig 4 : dividing corner area

We then compute, for each of these 9 areas, mean value and standard deviation for the 3 color planes, which gives us a vector of 54 attributes per corner.

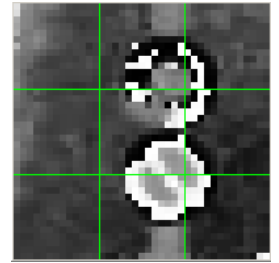

Hue plane

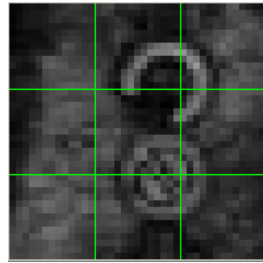

Sat. plane

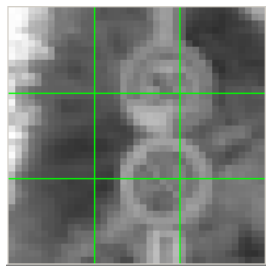

Lum. plane
Fig 5 : example of the 3 color planes for a traffic sign

For each candidate match, we build a 54 elements vector, made of the difference of the two vectors of the two corners involved. Before using this data, we must normalize it: each vector element $i$ of each candidate match $j$ is modified using the following expression :

$$
x_{i j}=\frac{x_{i j}-x_{i \min }}{x_{i \max }-x_{i \min }}
$$

with:

- $x_{i \max }$ : maximum value of the $i$ element for all the candidate matches

- $x_{i \text { min }}$ : minimum value of the $i$ element for all the candidate matches
This ensures that all elements of the vector have a value between 0 an 1 , and therefore have an equal weight in the vector. After normalizing, we compute and store the norm of this vector for each candidate match $j$, which is defined as :

$$
I_{j}=\sqrt{\sum_{i} x_{i j}}
$$

We can then sort the candidates list in increasing order using the vector norm, and start an iterative process, to extract valid pairs. The algorithm outline is presented below:

do \{

- find the first candidate match whose corners are not already used, and add it to the final pairs list

- discard all the candidate matches that use its left or right corner (unicity constraint)

\} while( a new pair has been found )

The major issue with this algorithm is that it will add to the final pairs list matches that have a high norm value, and that are obviously outliers. So we introduce a "stop" condition, that is related to the image characteristics. A good criterion is the current candidate match correlation score. So the loop condition of the above algorithm is modified as :

while \{

- a new pair has been found

AND - correlation of the current pair is below a threshold th \}

The threshold th is function of the average value of the candidate matches :

$$
t h=K_{c s} \frac{\sum_{i} c s_{i}}{n}
$$

with:

- $\mathrm{cs}_{\mathrm{i}}$ : candidate match correlation score (using ZNSSD criterion)

- $\mathrm{n}$ : number of candidate matches

- $\mathrm{K}_{\mathrm{cs}}$ : a parameter adjusted experimentally

\section{COMPUTING THE FUNDAMENTAL MATRIX}

At this point, we have obtained a list of matches, that we can use to recover the epipolar geometry, i.e. the Fundamental Matrix. Although these methods are designed to remove the maximum number of outliers, these are practically unavoidable. It has been shown [11] that the only methods that are able to give good results with the presence of outliers, are the "Random Sample Consensus" (RANSAC) method and the "Least Median of Squares" (LMEDS) method.

Both of these methods require a supplied error parameter, witch reflects the "a priori" knowledge of the precision of the expected estimation. Every data point that does not fit is then 
left unused by the algorithm, and tagged as rejected. We have experimented an iterative process to refine the computed matrix, by gradually removing points that had an epipolar distance higher than a self-adaptive threshold. This approach turned out to be deceptive, points far from image center were easily discarded, as their epipolar distance is naturally higher, regardless of their correctness. Far better results are achieved using the "straight" method, establishing the error parameter to a value between 0.5 and 0.8 pixels.

\section{ESTIMATING FUNDAMENTAL MATRIX ACCURACY}

In order to be able to compare the results to the correct geometry, we defined manually for each image pair a set of corresponding points. These points are used to compute a reference fundamental matrix $F_{R}$, that will be used as a ground truth for comparison with the estimated Fundamental matrix. $F_{R}$ is computed using the classical "8 points" method. This method gives the best results, as the corresponding points are outlier free. In order to improve reliability, the references points must spread over the whole image. In our experiments, we used between 10 to 15 manually defined points.

In order to have an estimation of the uncertainty of this reference matrix, one can find a rigorous approach in [17], but this method demands a great number of points, and therefore isn't appropriate in our case. We defined a simpler "accuracy factor", that indicates how accurate this reference Fundamental matrix $F_{R}$ is, related to the provided corresponding points. This factor is the average epipolar distance, for all corresponding points. This distance is defined as the distance between a point and the line generated by the opposite correspondent point in the other image.

For each image point $\boldsymbol{m}=[\mathbf{u}, \mathbf{v}, \mathbf{1}]$, the corresponding epipolar line $\boldsymbol{l}^{\prime}=\left[\mathbf{a}^{\prime}, \mathbf{b}^{\prime}, \mathbf{c}^{\prime}\right]$ in the other image is defined as:

$$
l^{\prime}=F_{R} \cdot m
$$

The absolute distance between this line and the right pair point $\boldsymbol{m}^{\prime}=\left[\boldsymbol{u}^{\prime}, \boldsymbol{v}^{\prime}, \boldsymbol{1}\right]$ is given by:

$$
\text { dist }=\left|\frac{a^{\prime} u^{\prime}+b^{\prime} v^{\prime}+c^{\prime}}{\sqrt{a^{\prime 2}+b^{\prime 2}}}\right|
$$

These expressions are similar for the left image. If $n$ correspondent points are given, the accuracy factor is the average distance of the $2 \mathrm{n}$ distances. It must be noted that this only gives an indication of how the given points "fit" together, and can not be considered as an indication of correctness of the reference Fundamental matrix. However, as one can assume that the provided points are outlier-free, it gives an indication of accuracy of the matrix. In our experiments, we achieved a value between 0.2 and 0.8 pixels.

This reference Fundamental matrix $F_{R}$ is used to compute a criterion of reliability of the estimated Fundamental Matrix
$\mathrm{F}$, defined as the euclidean distance in pixels between these two matrices. We used the "Laveau" method, as described in [5], p.184. It is based on the average distance between a random point and the corresponding epipolar line, while sampling the whole image space. In our experiments, we used 50000 samples.

\section{EXPERIMENTAL RESULTS AND DISCUSSION}

We developed software that implements the algorithms described here, and processed several road image pairs, but we present here only two, that are representative of the difficulties that a real system will encounter. Test images were 640 x 480 pixels wide, issued from a basic CCD camera, and are of an unknown geometry. However, they are similar in orientation and baseline to what would have been acquired with an embedded road vision system (baseline is approximately $1 \mathrm{~m}$.). The ROI is shown in light blue (the bottom part of images has been removed).

\section{A - Experimental data used}
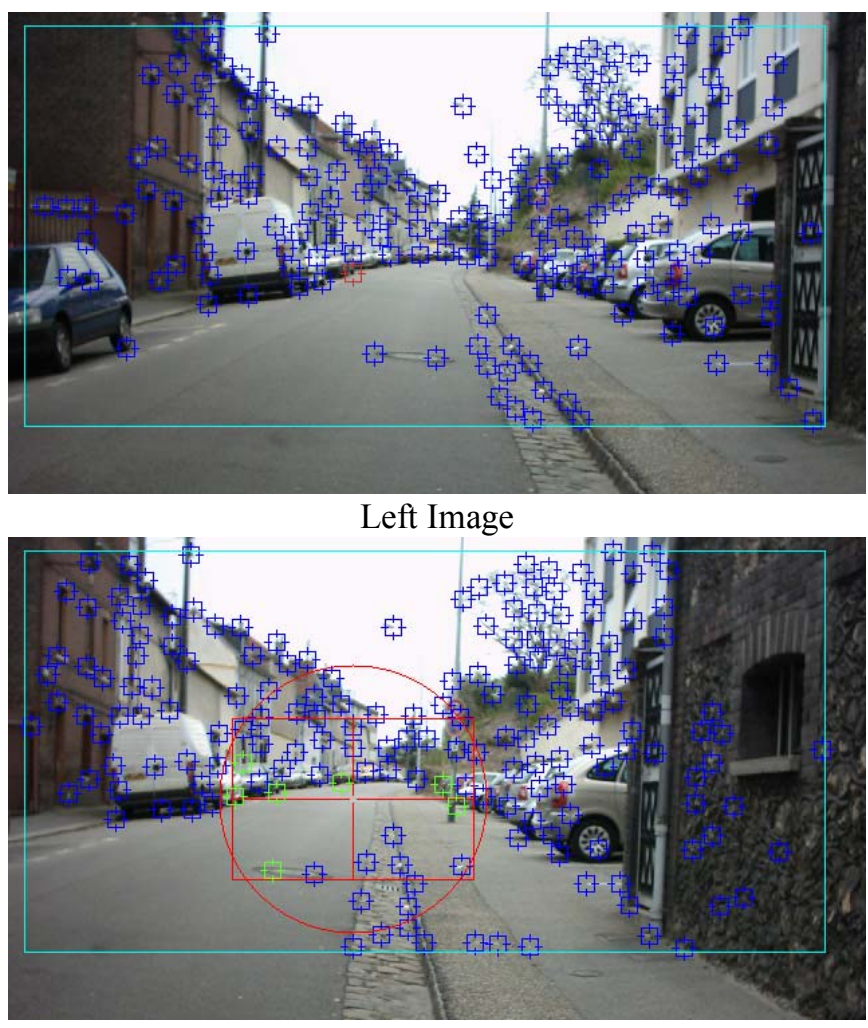

Right Image

Fig. 6 : scene 1 candidate matches

The first image pair shown on fig. 6 with the corners involved in the candidate match list, is a typical "street" scene, where the sky appears as a uniformly white area, and color variety is rather poor. Many structural elements (cars, buildings,...) can be observed. 
On the right image, the search area is shown in red, and the green interest points are those that are potential matches for the one shown in red in the left image.

The second image pair (fig. 7) represents a much more difficult case: many interest points are located in trees, and there are not many structural elements in the scene. As one can see by the selected left corner and its possible matches in the right image, establishing a correlation-based relationship is not easy in such an image, since many similar points are candidates.
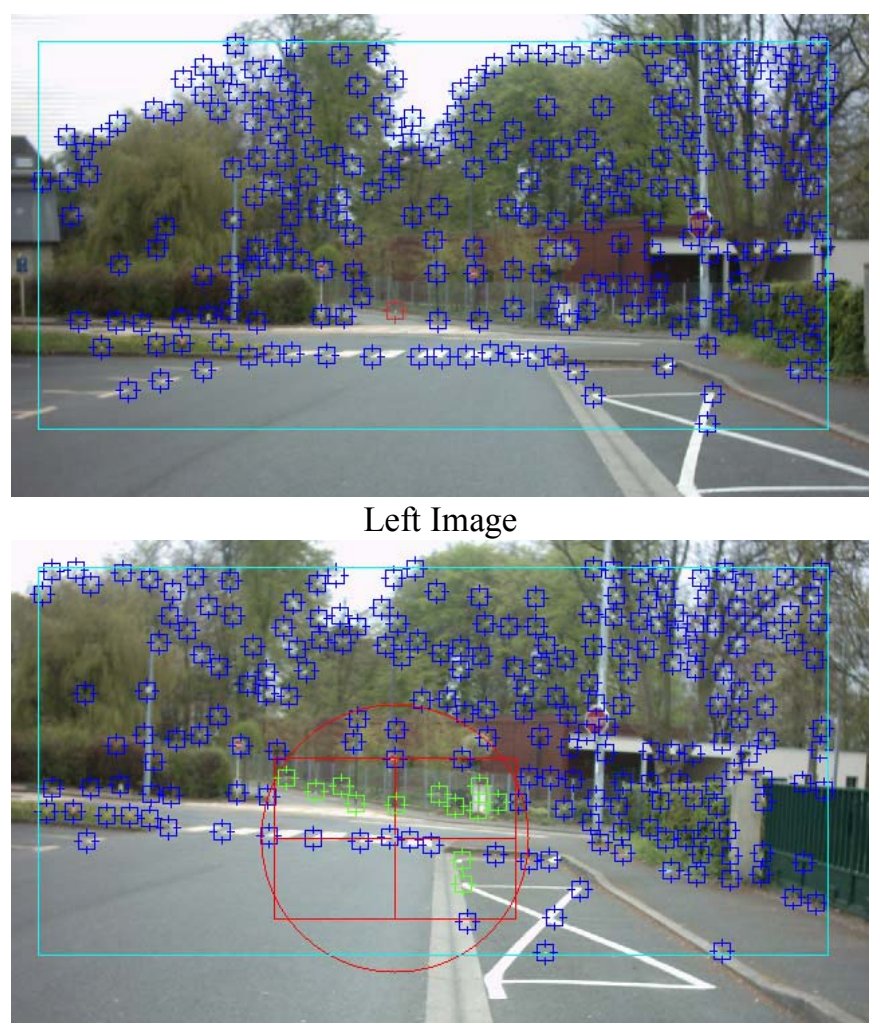

Right Image

Fig. 7 : scene 2 candidate matches

These sets of candidates were obtained with thresholds of 0.23 for the ZNSSD correlation criterion, and 0.45 for the ZNCC criterion. The first image pair generates 1104 candidate matches, and the second 1686.

For each image pairs, reference points have been manually set, and the associated Fundamental matrix computed. We achieved for the first image pair an accuracy factor of 0.185 pixels, and 0.081 for the second.

\section{$B$-Results}

The performance criterion is the distance between the reference Fundamental matrix and the estimated one using our method, while varying the only parameter accessible at the matching phase, that is the Kcs coefficient, and this for both LMEDS and RANSAC computed Fundamental matrices. We also provide the number of matches computed with our method, and the number of matches actually used by the LMEDS/RANSAC method. Results are shown in tables 1 and 2. These values are obtained using a "precision factor" of 0.7 pixels for the LMEDS/RANSAC algorithms, we measured it for each line of the result tables, and it actually never exceeded 0.3 pixels.

\begin{tabular}{|c|c|c|c|c|c|}
\hline & & \multicolumn{2}{|c|}{ LMEDS } & \multicolumn{2}{c|}{ RANSAC } \\
\hline $\mathrm{K}_{\mathrm{cs}}$ & $\begin{array}{c}\text { Nb of } \\
\text { matches }\end{array}$ & $\begin{array}{c}\text { matches } \\
\text { used }\end{array}$ & $\mathrm{d}$ (pixels) & $\begin{array}{c}\text { matches } \\
\text { used }\end{array}$ & $\mathrm{d}$ (pixels) \\
\hline 0.4 & 15 & 8 & 122.4 & 12 & 45.2 \\
\hline 0.65 & 28 & 18 & 25.5 & 19 & 12.4 \\
\hline 0.8 & 55 & 32 & 12.5 & 32 & 12.5 \\
\hline 0.9 & 71 & 31 & 28.5 & 33 & 27.2 \\
\hline 1.65 & 116 & 28 & 6.3 & 38 & 18.2 \\
\hline 1.8 & 130 & 34 & 36.7 & 35 & 20.2 \\
\hline
\end{tabular}

Table 1 : results for scene 1

\begin{tabular}{|c|c|c|c|c|c|}
\hline & & \multicolumn{2}{|c|}{ LMEDS } & \multicolumn{2}{c|}{ RANSAC } \\
\hline $\mathrm{K}_{\mathrm{cs}}$ & $\begin{array}{c}\text { Nb of } \\
\text { matches }\end{array}$ & $\begin{array}{c}\text { matches } \\
\text { used }\end{array}$ & $\mathrm{d}$ (pixels) & $\begin{array}{c}\text { matches } \\
\text { used }\end{array}$ & $\mathrm{d}$ (pixels) \\
\hline 0.55 & 15 & 11 & 9.1 & 14 & 10.5 \\
\hline 0.6 & 47 & 26 & 22.4 & 29 & 21.7 \\
\hline 0.65 & 52 & 28 & 72.3 & 31 & 19.9 \\
\hline 0.85 & 68 & 29 & 27.3 & 33 & 36.8 \\
\hline 1.2 & 82 & 27 & 11.4 & 35 & 42.2 \\
\hline 1.5 & 113 & 38 & 38.4 & 40 & 82.1 \\
\hline
\end{tabular}

Table 2 : results for scene 2

\section{C-Discussion}

While results can be considered in certain conditions as acceptable, it appears that the parameter has unpredictable influence on the accuracy, so it is difficult to give the "right" value. However, it appears clearly that there seems to be an "ideal" number of matches where the accuracy is highest: except for the first line of table 1 (which we consider as coincidence), below 20-30 matches, it is highly probable that accuracy will be bad, and if more than a hundred, the probability of having an important number of outliers, that will depreciate accuracy, is important.

It must be also told that this method is rather fast, as the sole matching process takes less than $300 \mathrm{~ms}$ for a hundred matches on a regular laptop. This is a valuable characteristic, particularly compared with the "Zhang" method, that is far much slower.

\section{CONCLUSION}

We have presented a new method for automatically estimating the epipolar geometry of a road color vision system. While results are encouraging, giving a fairly correct estimation, accuracy still needs to be improved. Particularly, parameters influence lacks stability. Further experiments will include integrating color information into the correlationbased list construction, and working with synthetic images to 
refine the accuracy results. It is also planned to use data from a video stream, in order to use the previous estimation of Fundamental matrix to reduce the amount of outliers.

\section{REFERENCES}

[1] G. Toulminet, S. Mousset, A. Bensrhair, "Fast and Accurate stereo vision based estimation of $3 \mathrm{~d}$ position and axial motion of road vehicles", in International Journal of Image and Graphics, Vol. 4, No. 1 (2004), 127

[2] J. Rebut, G. Toulminet A. Bensrhair, "Road Obstacles Detection using a Self-Adaptive Stereo Vision Sensor: a contribution to the ARCOS french project", in Proceedings of IEEE Intelligent Vehicle Symposium, june 2004, Parma, 738-743

[3] R. Labayrade, D. Aubert, J.P. Tarel, "Real Time Obstacle Detection in Stereovision on non Flat Road Geometry Through "V-disparity" representation", in Proceedings of IEEE Intelligent Vehicle Symposium, june 2002, Versailles, France

[4] C. Loop, Z. Zhang, "Computing rectifying homographies for stereo vision", in Proceedings of IEEE Computer Vision and Pattern Recognition, june 1999, Fort Collins, Colorado, USA, pp.125-131

[5] Zhang Z., "Determining the Epipolar Geometry and its Uncertainty: A Review", ", in International Journal of Computer Vision, Vol. 27, No 2 (1998), pp. 161-195

[6] Q.T. Luong, O. Faugeras, "The Fundamental Matrix: theory, algorithms, and stability analysis", in International Journal of Computer Vision, janv-96, Vol 17, Issue 1, 43-75

[7] R.I. Hartley and A. Zisserman, "Multiple View Geometry in Computer Vision", Cambridge Univ. Press, June 2000

[8] John Mallon, Paul F. Whelan, "Projective rectification from the fundamental matrix", in Image and Vision Computing, Vol. 23 (2005), pp. 643-650
[9] D.T. Oram, "Rectification for Any Epipolar Geometry", in Proceedings of British Machine Vision Conference, 2001

[10] P.R.S Mendonca, R. Cipolla, "A simple technique for self calibration", Proceedings of IEEE Computer Vision and Pattern Recognition, june 1999, Fort Collins, Colorado, USA, pp. 1500-1506

[11] Zhang Z., Deriche R., Faugeras O., "A Robust Technique for Matching two Uncalibrated Images Through the Recovery of the Unknown Epipolar Geometry", in Artificial Intelligence Journal, Vol. 78, Issue 1-2, 87-119, and also RR2273, INRIA-France

[12] S. Kramm, P. Miché, A. Bensrhair, "Self calibration of a road stereo vision system through correlation criterions", in Proceedings of IEEE Intelligent Vehicle Symposium, Tokyo, 2006, to appear.

[13] R. Zabih and J. Woodfill., "Non-parametric local transforms for computing visual correspondance", in Proceedings of the 3rd European Conference on Computer Vision, Stockholm, Sweden, pp. 151-158, May 1994

[14] Z.D. Lan, R. Mohr, Robust Location based Partial Correlation, RR3186, INRIA, France

[15] C. Harris, M. Stephens, "A combined Corner and Edge detector", in Proceedings of 4th Alvey Conference, pp. 147-151, 1988

[16] Intel OpenCV vision library, http://www.intel.com/technology/computing/opencv/ind ex.htm

[17] Csurka G., Zeller C., Zhang Z. and Faugeras O. "Characterizing the uncertainty of the fundamental matrix", in Computer Vision and Image Understanding, 68(1):18-36, 1997

[18] Lowe D. G., "Distinctive image features from scaleinvariant keypoints," in International Journal of Computer Vision, 60, 2 (2004), pp. 91-110 